\title{
Mortality Trend for Tumor Correlated Immune System in Hyperendemic Area of HCV Infection in Southern Italy: Joinpoint Analysis
}

\author{
Maurizio Montella ${ }^{1,}$, , Matteo Malvezzi ${ }^{2,3}$, Maria Grimaldi ${ }^{1}$, Flavia Nocerino ${ }^{1}$, Ferdinando \\ Frigeri ${ }^{4}$, Antonio Pinto ${ }^{4}$, Aldo Giudice ${ }^{1}$, Anna Crispo ${ }^{1}$ \\ 1 Epidemiology Unit, Istituto Nazionale per lo Studio e la Cura dei Tumori “Fondazione Giovanni Pascale”, IRCCS, Naples, Italy \\ 2 Department of Epidemiology, "Mario Negri" Farmachological Research Institute, IRCCS, Milan, Italy \\ 3 Department of Clinical Sciences and Community Health, Università degli Studi di Milan, Milan, Italy \\ 4 Hematology Unit, Istituto Nazionale per lo Studio e la Cura dei Tumori “Fondazione Giovanni Pascale", IRCCS, Naples, Italy \\ *Corresponding author: Maurizio Montella, Epidemiology Unit, Istituto Nazionale per lo Studio e la Cura dei Tumori “Fondazione Giovanni Pascale”, IRCCS, Naples, Italy. Tel: +39- \\ 815903816, Fax: +39-815462900, E-mail: epidemiologia.int@alice.it.
}

Received: Jun 06, 2013; Revised: Jun 20, 2013; Accepted: July 20, 2013

\begin{abstract}
Background: In many regions of southern Italy, hepatitis C virus (HCV) infection represents a major health problem (with a prevalence rate between $6 \%$ and $13 \%$ ). HCV is associated with different kinds of neoplasms such as non-Hodgkin lymphomas (NHL), and with autoimmune diseases (cryoglobulinemia), which develop after the virus has caused immune system alterations.

Objectives: To provide updated information on trends in mortality in a major metropolitan area of southern Italy from NHL, multiple myeloma and Hodgkin disease we analyzed cancer mortality data from 1988 to 2009.

Materials and Methods: Mortality data were extracted from National death certificates by age groups, gender, residence and cause of death by the Italian national institute of statistics (ISTAT). Age-standardized mortality rates (SMR) were computed applying the direct method and using the world standard population. To quantify the recent direction of temporal trends in older populations over time, truncated age-adjusted mortality rates were calculated for people aged 65 years and older. Cancer mortality trends were described using their estimated annual percent change (EAPC) and related 95\% Confidence Interval (CI).

Results: Statistically significant increasing EAPC was found among women for NHL $(+2.0 \%$ / year), while statistically significant decrease was found among men and women for $\mathrm{HD}(-3.5 \%$ / year, $-3.4 \%$ / year, respectively). No statistically significant EAPC was found for multiple myeloma.

Conclusions: The association between viral hepatitis and NHL in the area of interest might provide some degree of explanation to this finding. Our data confirm that due to epidemic infection of HCV in the area of Naples, a high mortality for NHL persists, moreover the adoption of standard therapeutic protocols administered in full accordance with an evidence-based approach and current guidelines explain reduced mortality from Hodgkin lymphomas.
\end{abstract}

Keywords: Hepacivirus; Lymphoma, Non-Hodgkin; Mortality; Analysis

\section{Background}

In many regions of southern Italy, hepatitis $\mathrm{C}$ virus (HCV) infection represents a major health problem (with a prevalence rate between 6 and 13\%) (1). In the past year the factors that brought about the prevalence of HCV were the extensive use of glass syringes, poor education and promiscuous poverty. Moreover sanitary procedures (surgical and dental interventions) and the health system in general are still less efficient and less meticulous than in Northern Europe and in the rest of Italy (2). Infection with hepatitis B virus (HBV) and hepatitis C virus (HCV) is a major risk factor for HCC in developed countries, but HCV is associated with different kinds of neoplasms such as non-Hodgkin lymphomas (NHL), and with autoimmune diseases (cryoglobulinemia), which develop af- ter the virus has caused immune system alterations (3-6). An association with multiple myelomas has been noted, while no association has been shown for Hodgkin disease $(7,8)$. HCV is an RNA virus that cannot be integrated with the host genome; it can, however, exert its oncogenetic potential indirectly by contributing to the modulator effects of the host immune system, probably through a capacity to elude the immune system (9).

\section{Objectives}

To provide updated information on trends in mortality in a major metropolitan area of southern Italy (Naples 3,500,000 inhabitants) from non-Hodgkin lymphoma, multiple myeloma and Hodgkin disease we analyzed cancer mortality data for all ages and for 65+ truncated age

Implication for health policy/practice/research/medical education:

We believe that our study is particular relevant because in many regions of Southern Italy, hepatitis C virus (HCV) infection represents a major health problem (with a prevalence rate between $6 \%$ and $13 \%$ ); for the first time we provide updated information on trends in mortality in a metropolitan area of southern Italy for some tumors correlated to HCV.

Copyright (C) 2013, KowsarCorp.; Licensee Kowsar Ltd. This is an Open Access article distributed under the terms of the Creative Commons Attribution License(http:/ creativecommons.org/licenses/by/3.0), which permits unrestricted use, distribution, and reproduction in any medium, provided the original work is properly cited. 
group from 1988 to 2009 .

\section{Materials and Methods}

Mortality data were extracted from National death certificates by age ( 5 years) groups, gender, residence and cause of death. These records were made available by the Italian national institute of statistics (ISTAT). The time window considered spanned from 1988 to 2009. International classification of diseases (ICD-9 and ICD-10) changed twice from 1998 to 2008 therefore records related to cancer deaths were re-coded according to the tenth revision of the ICD.

\subsection{Data Analysis}

From the matrices of certified deaths and resident population we extracted mortality data related to 5-year agegroups for every calendar year between 1988 and 2009. Data for the years 2004 and 2005 were not available.

\subsection{Statistical Analysis}

Age-standardized mortality rates (SMR) were computed for each 5-year age group, by gender, primitive cancer site and province applying the direct method and using the world standard population. To quantify the recent direction of temporal trends in older populations over time, truncated age-adjusted mortality rates were calculated for people aged 65 years and older. Cancer mortality trends between 1988 and 2009 were analyzed using joinpoint regression, using the program provided by the United States surveillance epidemiology and end results (US SEER) (10). Cancer mortality trends were described using their estimated annual percent change (EAPC) and related $95 \%$ confidence interval $(\mathrm{CI})$.

\section{Results}

For all ages between 1988 and 2009, in men from Naples, SMR for non-Hodgkin lymphoma increased from 3.6 to 4.2 per 100,000 among women, SMRs increased slightly from 2.0 to 2.4 per 100.000 . SMRs for multiple myeloma, in the study period, stay the same in both sexes (from 1.1 to 1.7 per 100,000 and from 1.6 and 1.5 per 100,000 in men and women respectively). Whereas for Hodgkin disease during the period 1988 to 2009 decreased SMRs were observed either for men or women (from 0.9 to 0.4 per 100,000 and 0.8 to 0.4 per 100,000 respectively). In the $65+$ truncated age group from Naples, SMRs for nonHodgkin lymphoma increased from 20.0 to 26.6 per 100.000 in men and from 8.7 to 16.0 per 100.000 in women. Similarly to the results of all ages in the elderly for multiple myeloma SMRs were uniform during the study period in both sexes (from 11.2 to 16.6 per 100,000 and 15.3 to 15.8 per 100,000 in men and women respectively). For Hodgkin disease, decrease SMRs were observed for men and women (2.8 to 2.0 per 100.000 and from 3.4 to 1.5 per 100.000 , respectively).

Results from the joinpoint regression analysis are shown in Figure 1 for all ages and in Figure 2 for the elderly (65 over); EAPC values are given by sex for non-Hodgkin lymphoma, multiple myeloma and Hodgkin disease from 1998 to 2009.

Figure 1. All Ages Joinpoint Analysis for NHL, Multiple Myeloma, Hodgkin's Disease in the Metropolitan Area of Naples According to Gender, From 1988 to 2009
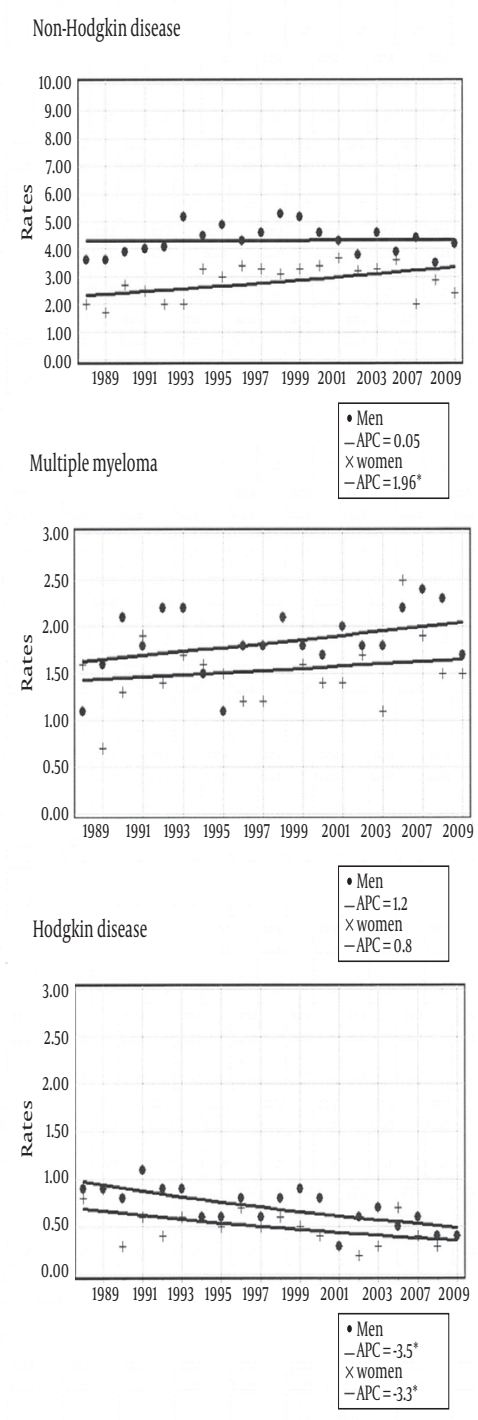

Men data points (circle), men joinpoint model (solid line). Women data points (cross), women joinpoint model (solid line) 
Figure 2. 65+ Truncated Age-Group Joinpoint Analysis of Age-Standardized Death Rates for NHL, Multiple Myeloma, Hodgkin's Disease in the Metropolitan Area of Naples According to Gender, From 1988 to 2009

Non-Hodgkin disease 65t

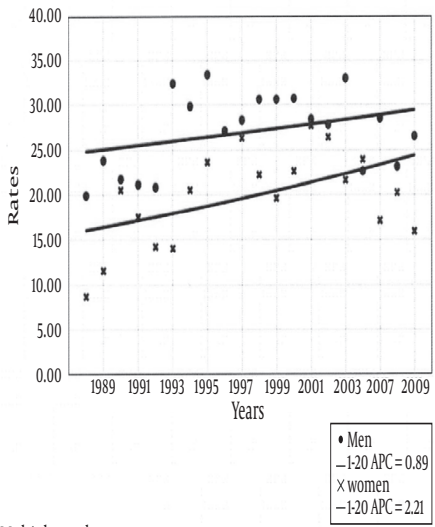

Multiple myeloma $65+$
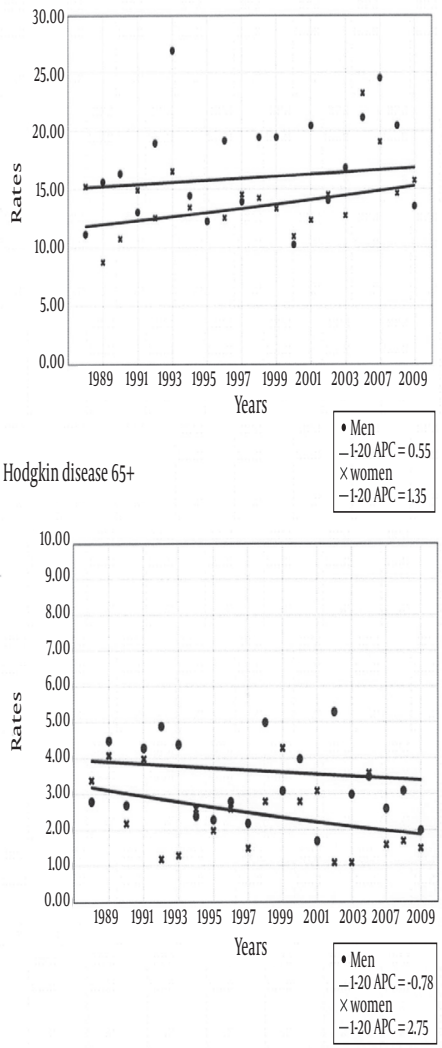

Men data points (circle), men joinpoint model (solid line). Women dat points (cross), women joinpoint model (solid line)
Statistically significant increasing EAPC was found among women for NHL (+2.0\% / year), while statistically significant decrease was found among men and women for $\mathrm{HD}(-3.5 \%$ / year, $-3.4 \%$ / year, respectively). No statistically significant EAPC ware found for multiple myeloma. The 65+ truncate age group had no significant NHL increasing EAPC both in men $(\mathrm{EAPC}=+0.01 \%$ year, $\mathrm{EAPC}=+0.89 \% /$ year; respectively), and in women (EAPC $=+1.96 \% /$ year, $\mathrm{EAPC}=+2.21 \% /$ year; respectively). For multiple myeloma the rise was only observed in women for all ages, finally for Hodgkin disease the negative EAPC values were smaller in the elderly compared to the all ages group both in men (EAPC $=-3.5 \%$ | year, EAPC $=-0.78 \%$ (year; respectively) and in women $($ EAPC $=-3.4 \%$ year, EAPC $=-2.75 \%$ / year; respectively $)$.

\section{Discussion}

Our results for non-Hodgkin lymphoma are not consistent with the decrease in mortality seen in the Italian data $(\mathrm{APC}=-3.1 \% /$ year in men and $\mathrm{APC}=-3.9 \% /$ year in women; $\mathrm{APC}=-1.4 \% /$ year in men and $\mathrm{APC}=-1.7 \%$ | year in women over 65 years) (11). We observed significantly increasing cancer mortality trends from nonHodgkin lymphoma in women from the metropolitan area of Naples, which were somewhat consistent with the non-significant increases reported for females in elderly women aged 65 and older. The available evidence on the association between viral hepatitis and non-Hodgkin lymphoma in the metropolitan area of interest might provide some degree of explanation to this finding. Indeed, in a previous hospital-based casecontrol study including participants from Naples, $\mathrm{HCV}$ infection was associated with a significantly increased risk of non-Hodgkin lymphoma, with the fraction of cases attributable to HCV being $12.4 \%$ (7) These results were consistent with those from a previous study including people from Campania and assessing the association of HCV with a number of tumors correlated with the immune system. HCV infection was associated with greater risk not only for B-cell nonHodgkin lymphoma, but also for multiple myeloma (odds ratios (OR) 3.7 and 95\% CI 1.9-7.4 and OR 4.5, and 95\% CI 1.9 - 10.7, respectively) $(8,12)$.

A possible explanation for this association could be the ability of HCV to infect early differentiation stages of hematopoietic cells. In this case, a subsequent mutational event, occurring before complete maturation, (e.g. before germinal center entry) gives an aggressive disease (13). The mechanisms responsible for persistence of viral infection and for the cellular lesion are not well understood. Despite an active response from the host, HCV has the capacity to elude the immune system. It is believed that the quasispecies nature of $\mathrm{HCV}$ is one of the major mechanisms allowing the vi- 
rus to develop chronic infection $(6,9)$.

Existing literature suggest that exposure to polychlorinatedbiphenyls (PCBs) contributes to NHL risk. In a recent study showed that polychlorinated biphenyls (PCBs) exposure can compromise the immune surveillance mechanism. Highly chlorinated PCNs with strong affinity for hydrocarbon receptor (Ahr) are potent immonotoxicants that increase NHL risk. All PCBs can induce reactive oxygen species formation, genotoxic effects, immune suppression and inflammatory response to various extents and through different pathways (14). Nevertheless the high prevalence of HCV in the Neapolitan area, may largely contribute to explain the higher mortality rate for NHL in our study $(1,7,8)$.

Our data confirm that due to epidemic infection of HCV in the area of Naples, a high mortality for NHL persists, moreover the adoption of standard therapeutic protocols and integrated treatments administered in full accordance with an evidence-based approach and current guidelines explain reduced mortality from Hodgkin lymphomas, especially in patients younger than 65 years of age.

\section{Acknowledgements}

We declare to have not acknowledgments for this manuscript.

\section{Author Contribution}

Study concept and design: Montella and Crispo. Analysis and interpretation of data: Montella, Crispo and Malvezzi. Drafting of the manuscript: Montella. Critical revision of the manuscript for important intellectual content: Frigeri and Pinto. Statistical analysis: Crispo. Finally Grimaldi and Nocerino contributed to input data and prepared the manuscript.

\section{Financial Disclosure}

We have no financial and personal relationships with other people or organizations that could inappropriately influence the work.

\section{Funding/Support}

This manuscript was not support by any sponsor.

\section{References}

1. Guadagnino V, Stroffolini T, Rapicetta M, Costantino A, Kondili LA, Menniti-Ippolito F, et al. Prevalence, risk factors, and genotype distribution of hepatitis $\mathrm{C}$ virus infection in the general population: a community-based survey in southern Italy. Нераtology. 1997;26(4):1006-11.

2. Montella M, Crispo A, Serraino D, Rezza G, Franceschi S. Is the spread of HCV in Southern Italy attributable to iatrogenic transmission through unsterile injections? Eur J Cancer Prev 2003;12(1):85-6.

3. Montella M, Crispo A, Giudice A. HCC, diet and metabolic factors: Diet and HCC. Hepat Mon. 2011;11(3):159-62.

4. Ohsawa M, Shingu N, Miwa H, Yoshihara H, Kubo M, Tsukuma H et al. Risk of non-Hodgkin's lymphoma in patients with hepatitis C virus infection. Int J Cancer. 1999;80(2):237-9.

5. Silvestri F, Pipan C, Barillari G, Zaja F, Fanin R, Infanti L, et al. Prevalence of hepatitis $C$ virus infection in patients with lymphoproliferative disorders. Blood.1996;87(10):4296-301.

6. Dammacco F, Gatti P, Sansonno D. Hepatitis C virus infection, mixed cryoglobulinemia, and non-Hodgkin's lymphoma: an emerging picture. Leuk Lymphoma. 1998;31(5-6):463-76.

7. Talamini R, Montella M, Crovatto M, Dal Maso L, Crispo A, Negri $\mathrm{E}$, et al. Non-Hodgkin's lymphoma and hepatitis $\mathrm{C}$ virus: a casecontrol study from northern and southern Italy. Int $J$ Cancer 2004;110(3):380-5.

8. Montella M, Crispo A, Frigeri F, Ronga D, Tridente V, De Marco $\mathrm{M}$, et al. HCV and tumors correlated with immune system: a case-control study in an area of hyperendemicity. Leukemia Res. 2001;25(9):775-81.

9. Farci P, Shimoda A, Coiana A, Diaz G, Peddis G, Melpolder JC, et al The outcome of acute hepatitis $C$ predicted by the evolution of the viral quasispecies. Science. 2000;288(5464):339-44

10. Joinpoint Regression Program: National Cancer Institute 2010.

11. Arfe A, Malvezzi M, Bertuccio P, Decarli A, La Vecchia C, Negri E. Cancer mortality trend analysis in Italy, 1970-2007. Eur J Cancer Prev. 2011;20(5):364-74.

12. Montella M, Crispo A, Russo F, Ronga D, Tridente V, Tamburini M. Hepatitis $C$ virus infection and new association with extrahepatic disease: multiple myeloma. Haematologica. 2000;85(8):883-4.

13. Dolcetti R, Boiocchi M. Cellular and molecular bases of B-cell clonal expansions. Clin Exp Rheumatol. 1996;14 Suppl 14:S3-13.

14. Lauby-Secretan B, Loomis D, Grosse Y, Ghissassi FE, Bouvard V, Benbrahim-Tallaa L, et al. Carcinogenicity of polychlorinated biphenyls and polybrominated biphenyls. Lancet Oncol. 2013;14(4):287-8. 